\title{
DESENVOLVIMENTO E VALIDAÇÃO DO APLICATIVO TEENSAÚDE®: UM INSTRUMENTO DE APOIO EDUCACIONAL EM SAÚDE PARA ADOLESCENTES
}

Italo Ricelly Braz, UNIVASF, italoricelly@hotmail.com, http://orcid.org/0000-0002-

$$
\text { 1286-0521 }
$$

Ricardo Argenton Ramos, UNIVASF, ricardo.aramos@univasf.edu.br, http://orcid.org/0000-0002-9688-719X

Adriana Gradela, UNIVASF, agradela@ @otmail.com, http://orcid.org/0000-0001-5560$\underline{6171}$

Resumo: Este trabalho teve como objetivo a criação e validação de um aplicativo para dispositivos móveis como instrumento de apoio no processo de educação em saúde para adolescentes. Tratou-se de um estudo com metodologia de desenvolvimento tecnológico leve-dura e dividida em duas etapas: a primeira consistiu na construção do aplicativo Teensaúde® como uma ferramenta de aprendizado, abordando diversas temáticas relacionadas à saúde do adolescente, através de telas interativas, interface abstrata com cores e imagens atraentes e uma linguagem de texto clara, baseada na caderneta do adolescente. A segunda etapa, referiu-se à validação do aplicativo por especialistas e pelo público-alvo, que responderam a um formulário online, contendo 17 questões, baseadas no Mobile App Rating Scal-MARS. Os dados foram analisados por mensuração do Índice de Validade de Conteúdo (IVC), considerando-se como ideal uma taxa de IVC $\geq 0,78$. O IVC global foi de 0,78 pelos especialistas e 0,84 pelo público-alvo, tendo sido ressalvados alguns pontos que serão posteriormente adaptados. Conclui-se que o aplicativo Teensaúde® pode ser utilizado como instrumento de educação em saúde para adolescentes, pois além de contribuir com a compreensão das informações desta temática, estimula o processo de autocuidado.

Palavras-chave: Educação em Saúde. Saúde do adolescente. Software.

\section{DEVELOPMENT AND VALIDATION OF THE TEENSAUDE APPLICATION: A HEALTH EDUCATION INSTRUMENT FOR ADOLESCENTS}

\begin{abstract}
This work aimed to create and validate an application for mobile devices as a support tool in the health education process for adolescents. It was a study with a lighthard technology development methodology and divided into two stages: the first consisted of building the Teensaúde ${ }^{\circledR}$ application as a learning tool, addressing various topics related to adolescent health, through interactive screens, interface abstract with attractive colors and images and a clear text language based on the teenager's notebook. The second stage referred to the validation of the application by experts and the target audience, who responded to an online form, containing 17 questions, based on the Mobile App Rating Scal-MARS. Data were analyzed by measuring the Content Validity Index (CVI), considering an ideal rate of CVI > 0.78. The global CVI was 0.78 for the specialists and 0.84 for the target audience, with some points that will be adapted later. It is concluded that the Teensaúde ${ }^{\circledR}$ application can be used as a health education tool for adolescents, as it contributes to the understanding of information on this topic, as it encourages the self-care process.
\end{abstract}

Keywords: Health Education. Teen health. Software. 


\section{Introdução}

A adolescência é caracterizada como a fase de transição entre a infância e a vida adulta, sendo marcada por profundas mudanças físicas, psicológicas, sociais e comportamentais. De acordo com a Organização Mundial da Saúde (OMS), corresponde ao período entre 10 e 19 anos e se caracteriza pela necessidade de integração social; busca e desenvolvimento da personalidade; definição da identidade sexual; descoberta das próprias limitações; crescimento emocional e intelectual, aumento das relações interpessoais; vivência da afetividade e da sexualidade (SOARES et al., 2008). Em paralelo há neste período o crescimento da autonomia e da independência em relação à família, assim como a experimentação de novos comportamentos e vivências, os quais podem se tornar fatores de risco para a saúde como, por exemplo, o sexo desprotegido, a alimentação inadequada, o sedentarismo, o uso de drogas lícitas e/ou ilícitas e a negligência com a saúde bucal (MALTA et al., 2010). Assim, fazer o uso da educação abordando essas temáticas torna-se algo primordial e necessário.

Um dos caminhos viáveis para atingir uma grande quantidade de adolescentes é o uso das Tecnologias da Informação e Comunicação (TIC), pois são capazes de ampliar o acesso à informação utilizando como instrumentos o computador, a internet e dispositivos móveis (celulares, tablets etc.) (PINTO et al., 2017; FERREIRA; GOMES JUNIOR, 2021). Assim, através da integração de múltiplas mídias, linguagens e recursos, as TICs têm possibilitado o desenvolvimento de um processo educacional interativo (PINTO et al., 2017), contribuindo por mudar a forma como pacientes enfrentam suas condições nas doenças crônicas, promovendo o relacionamento com os profissionais de saúde e o autocuidado (BARRA et al., 2017; FERREIRA; GOMES JUNIOR, 2021).

Neste sentido, as novas tecnologias móveis podem se tornar uma poderosa ferramenta no processo ensino-aprendizagem de adolescentes, pois têm alcançado espaço significativo em suas vidas, pois $80 \%$ dos usuários da internet no Brasil são crianças e adolescentes (CGI - CETIC, 2015). Assim, a inserção das TIC na adolescência pode estimular a integração e os debates sobre sua saúde, bem como a disseminação de informações sobre o tema de maneira lúdica e, ao mesmo tempo, séria e aplicável (VENTOLA, 2014) além de estimular o autocuidado (SOUZA, 2013). Além disso, estudos recentes com adolescentes inseridos em diversos espaços sociais demonstraram que as novas TIC são úteis para se trabalhar as temáticas transversais com a saúde do adolescente (TORRES et al., 2015).

O papel das novas TIC como ferramentas importantes no processo de ensinoaprendizagem de adolescentes somado a carência de aplicativos voltados à promoção de sua saúde, tornam necessários estudos que busquem a construção de aplicativos para dispositivos móveis com esta finalidade, particularmente, se estes puderem ser utilizados em escolas, Unidades de Saúde da Família e outros espaços sociais. Diante disso, essa pesquisa teve como objetivo desenvolver e validar um aplicativo para dispositivos móveis como instrumento de apoio no processo de educação em saúde para adolescentes.

\section{Material e Métodos}

Esta pesquisa foi aprovada pelo Comitê de Ética em Pesquisa (CEP) da Universidade de Pernambuco (UPE) com o CAAE: 49990421.6.0000.5192 e desenvolvida em concordância com as Diretrizes e Normas de Pesquisa em seres Humanos preconizadas pela resolução 466/2012 do Conselho Nacional de saúde.

Tratou-se de um estudo metodológico de desenvolvimento de tecnologia leve dura, para obtenção de software de fácil utilização e aplicabilidade (POLIT; BECK, 2011), desenvolvido em duas etapas: a primeira consistiu na construção do aplicativo Teensaúde® e a segunda em sua validação. 
A etapa de construção seguiu os procedimentos descritos por Rossi (1996) e Zambalde et al. (1999) e seguiu quatro passos: modelagem, projeto de navegação, design abstrato da interface e implementação. No desenvolvimento do Teensaúde® foi utilizada a linguagem de programação Javascript, suportada pela Unity versão 5, pois é uma ferramenta de fácil uso, intuitiva e com interface gráfica simples, o que contribui para a organização dos arquivos do aplicativo (SILVA,2014).

$\mathrm{Na}$ etapa de validação foi considerado o quantitativo recomendado por Pasquali (1999), o qual poderia variar de 6 a 20. Assim, foram selecionados 10 especialistas, cinco na área da saúde, e cinco da área da informática e 10 adolescentes com idades entre 14 e 19 anos, de ambos os sexos, regularmente matriculados no Colégio Luiz Eduardo Magalhães, na cidade de Juazeiro/BA. Após o preenchimento online do Termo de Consentimento Livre e Esclarecido (TCLE), os avaliadores receberam o link de acesso ao aplicativo e, após manuseá-lo por 20 a 30 minutos, responderam ao questionário de avaliação Mobile App Rating Scal-MARS (STOYANOV et.al, 2015). Este instrumento era composto por 17 questões objetivas, com escalas de resposta partindo do ponto "Inadequado" e chegando até cinco pontos como "Excelente", e promovia a análise do aplicativo em quatro dimensões: envolvimento, funcionalidade, estética e informação. No final, foi acrescentado um espaço para comentários e sugestões de melhorias.

Os questionários foram analisados com base na mensuração do Índice de Validade de Conteúdo (IVC), muito utilizado na área da saúde por permitir a análise de cada item do instrumento e, posteriormente, sua análise como um todo (ALEXANDRE; COLUCI, 2011). Os resultados da gradação da escala de classificação MARS foram adaptados por seus respectivos pesos, conforme orienta Fehring (1987) em: 1) Inadequado $=0 ; 2$ ) Insuficiente $=0,25 ; 3)$ Aceitável $=0,50 ; 4)$ Muito bom $=0,75$ e 5) Excelente $=1 . \mathrm{Na}$ sequência foram organizados em um banco de dados para cálculo do IVC, segundo metodologia proposta por Polit e Beck (2011): IVC-1 (validade de conteúdo dos itens individuais) e IVC-2 (média dos índices de validação de conteúdo para todos os itens da dimensão), utilizando-se as seguintes fórmulas:

1)

IVC 1= Número de respostas "5" "4" "3" "2"

Número total de respostas

2)

IVC $2=\underline{\text { Soma de todas as respostas por itens }}$

Número total de respostas

Para cálculo do IVC global, os IVC's dos itens foram somados e divididos pelo número de itens, considerando-se como ideal um IVC superior a 0,78 (POLIT; BECK, 2006).

\section{Resultados e Discussões}

\subsection{Construção do aplicativo}

O nome do aplicativo surgiu da junção da palavra inglesa Teen, que significa adolescente, com a palavra saúde, pois o objetivo do mesmo era contribuir com a construção do conhecimento na área da saúde e estimular o autocuidado de adolescentes. Segundo Mano (2008), o interesse que os adolescentes têm por tecnologias produz automotivações que repercutem positivamente no aprendizado e em suas escolhas na trajetória de vida. Isso remete a necessidade de incorporá-las no ambiente escolar, no contexto familiar, nos serviços de saúde e nos diversos espaços comunitários.

A tela principal do Teensaúde® exibiu os principais temas da saúde do adolescente, como: adolescência, direitos, dicas de saúde, crescimento e desenvolvimento, saúde bucal, vacinação, puberdade, sexualidade, alimentação saudável; 


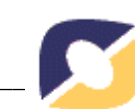

Infecções Sexualmente Transmissíveis (IST’s); Quiz e Informações sobre aplicativo (Figura 1).

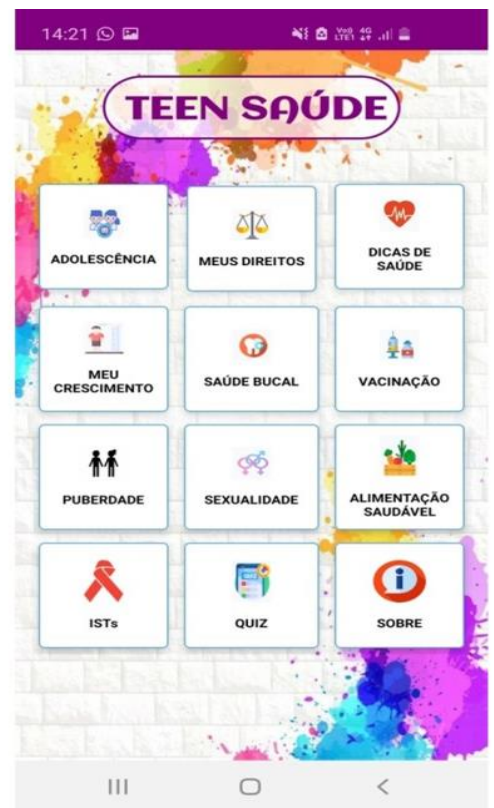

Figura 1- Tela principal do aplicativo Teensaúde®.

Estes temas foram baseados na caderneta de saúde do adolescente do Ministério da Saúde e em outros materiais pesquisados, os quais foram adaptados a uma linguagem acessível a eles, evitando-se o uso de termos técnicos específicos da área da saúde e procurando-se mesclar com imagens disponíveis na internet de forma gratuita. Wilkinson e Miller (2007) destacam que a qualidade e a adequação da linguagem e das ilustrações são aspectos considerados relevantes, pois uma tecnologia educativa de alta qualidade requer informações confiáveis e o uso de vocabulário claro, para permitir entendimento fácil do conteúdo.

Prensky (2012) ressalta que o uso de jogos digitais na educação pode tanto servir para aprendizagem desde o início, quanto para reforçar o conteúdo aprendido através dos métodos comuns de ensino. Um bom exemplo são o quiz, que estimulam a memorização, repetição e retenção de informações (MARTINS, 2017) como os utilizados no Teensaúde®.

Ferramentas interativas foram também utilizadas para despertar a atenção do público-alvo. Como exemplo, ao clicar em "Minhas Vacinas" no menu "Vacinação", podiam ser registradas as vacinas já recebidas e o aprazamento para as futuras vacinações (Figura 2).

A afirmação de Marques e Gallão (2020) de que as ferramentas de auxílio à educação possuem capacidade de facilitar a aprendizagem de determinado assunto e o alto uso da internet por adolescentes no mundo (LIVINGSTONE et al., 2016) e no Brasil (NIC.br, 2019), legitimam a importância de softwares educacionais como ferramentas pedagógicas auxiliares na promoção da aprendizagem. Neste sentido, o aplicativo Teensaúde ${ }^{\circledR}$ configurou-se como uma inovação tecnológica na área da saúde por ser um dos primeiros aplicativos educativos direcionados a saúde do adolescente. 

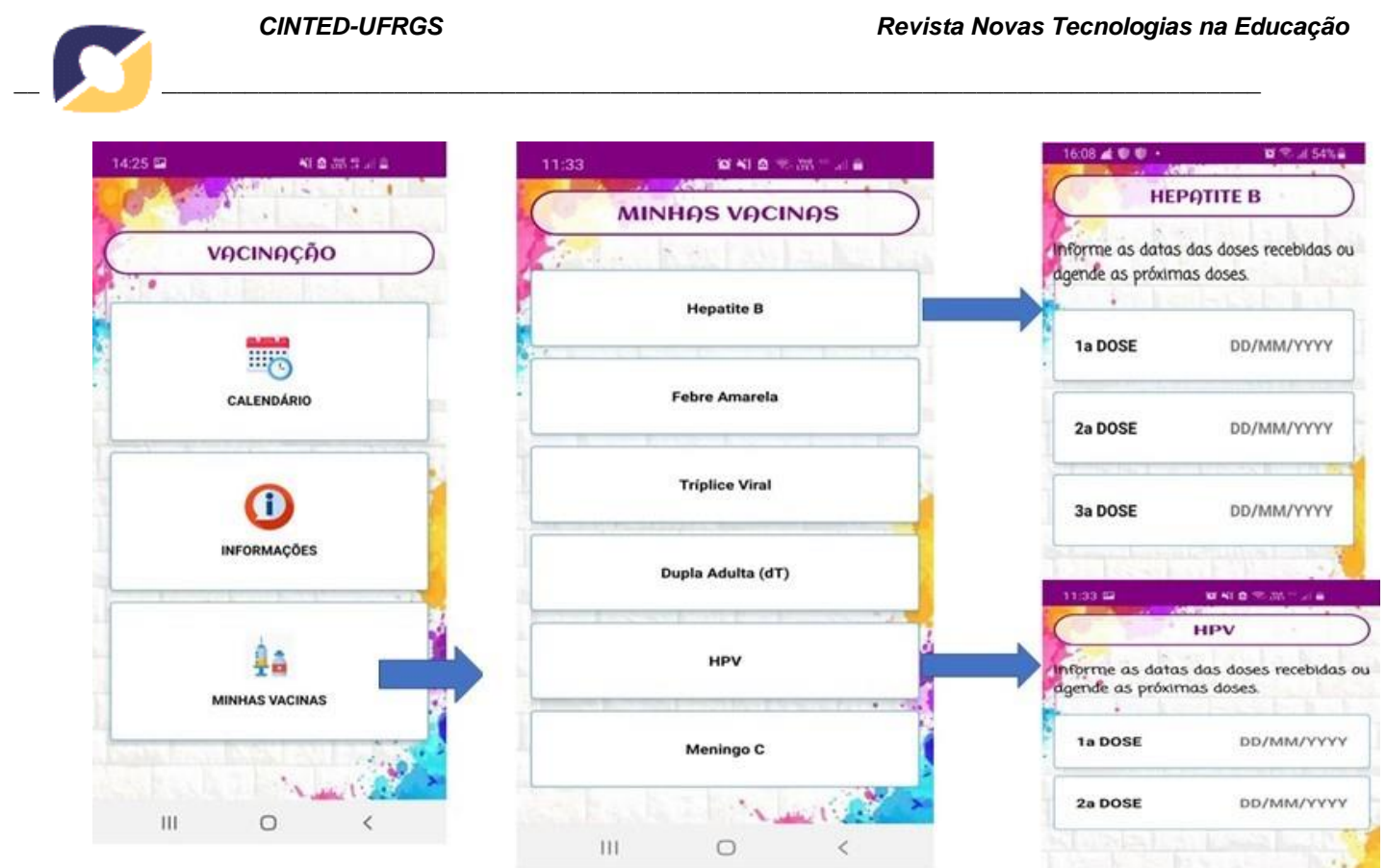

Figura 2: Imagem do submenu "Minhas vacinas" e da tela para registro da situação vacinal no aplicativo Teensaúde®.

Todavia, cabe ressaltar que o uso da internet nem sempre é positivo para os adolescentes, pois os expõem a muitos riscos on-line (LIVINGSTONE et al., 2017) que podem impactar sua vida cotidiana de modo desastroso à medida que influenciam sua educação, saúde física e mental, entretenimento, desenvolvimento sociocultural, vida política, entre outros aspectos (CÁNOVAS, 2015; LIVINGSTONE et al., 2017; SOUZA, 2019). Um outro aspecto negativo é que o uso intenso da internet pode levar ao isolamento social (LÉVY, 2010), causar alterações do funcionamento do cérebro (CARR, 2011) e até transtornos psiquiátricos (ROSEN et al., 2012).

Por isto é necessário atenção para que o uso das tecnologias não se torne excessivo e que o adolescente possa usufruir delas sem prejuízo de seu desenvolvimento social, cognitivo e emocional (SILVA; SILVA, 2017). Neste sentido sugestões como a mediação parental ativa; uso compartilhado da Internet (CABELLO et al., 2016); estabelecimento de regras sobre tempo e situações em que as mídias podem ser utilizadas (PREVITALE, 2006) são opções interessantes.

\subsection{Validação do aplicativo}

Para assegurar a valorização do desenvolvimento e o uso de um aplicativo faz-se necessário sua validação, na qual é de suma importância a avaliação de profissionais da área e do público-alvo, os quais podem colaborar com a melhoria da tecnologia construída. Na Tabela 1 foram apresentados os dados referentes a avaliação das quatro dimensões (envolvimento, funcionalidade, estética e informações) pelos especialistas.

A análise do envolvimento, funcionalidade e estética do aplicativo foi realizada pelos dois grupos de especialistas, totalizando 10 avaliações, enquanto a avaliação das informações contidas no aplicativo foi realizada apenas pelos especialistas da área da saúde $(n=5)$, devido ao conhecimento técnico deles direcionado as temáticas abordadas.

A dimensão "Envolvimento" apresentou o IVC2 abaixo do valor recomendado por Polit e Beck (2006), enquanto a dimensão "Funcionalidade" apresentou IVC2 mais alto. Os itens performance, facilidade de acesso, navegação e designer gestual obtiveram IVC's individuais superiores a 0,82 , que contribuíram para o alto IVC2 desta dimensão.

No quesito "Estética" foram avaliados os itens referentes ao layout, gráficos e identidade visual, tendo a categoria atingido valor um pouco abaixo do esperado (IVC2= 
0,70). Por outro lado, a dimensão "Informações" recebeu avaliação bastante satisfatória (IVC2 $=0,87$, indicando a boa qualidade e quantidade do conteúdo disponibilizado. A precisão da descrição e a explicação visual de conceitos, também apresentaram avaliação superior ao recomendado.

Tabela 1- Resultados da avaliação do aplicativo Teensaúde® pelos especialistas das áreas da saúde e da informática

\begin{tabular}{|c|c|c|c|c|c|c|}
\hline Análise & $\begin{array}{l}\text { Inade- } \\
\text { quado }\end{array}$ & $\begin{array}{l}\text { Insufici- } \\
\text { ente }\end{array}$ & Aceitá-vel & $\begin{array}{l}\text { Muito } \\
\text { Bom }\end{array}$ & $\begin{array}{c}\text { Excelen- } \\
\text { te }\end{array}$ & IVC1 \\
\hline \multicolumn{7}{|l|}{ ENVOLVIMENTO } \\
\hline Entretenimento & 0 & 0 & 4 & 5 & 1 & 0,65 \\
\hline Interesse & 0 & 0 & 2 & 4 & 3 & 0,7 \\
\hline Personalização & 1 & 1 & 4 & 3 & 1 & 0.51 \\
\hline Interação & 0 & 0 & 7 & 2 & 1 & 0,6 \\
\hline Grupo Alvo & 0 & 1 & 1 & 4 & 4 & 0,77 \\
\hline IVC2 & & & & & & $\mathbf{0 , 6 4}$ \\
\hline \multicolumn{7}{|l|}{ FUNCIONALIDADE } \\
\hline Performance & 0 & 01 & 0 & 04 & 05 & 0,82 \\
\hline Facilidade de acesso & 0 & 0 & 0 & 01 & 09 & 0,97 \\
\hline Navegação & 0 & 0 & 0 & 0 & 10 & 1,00 \\
\hline Designer gestual & 0 & 0 & 01 & 02 & 07 & 0,82 \\
\hline IVC2 & & & & & & $\mathbf{0 , 9 0}$ \\
\hline \multicolumn{7}{|l|}{ ESTÉTICA } \\
\hline Layout & 0 & 01 & 03 & 02 & 04 & 0,72 \\
\hline Gráficos & 0 & 01 & 03 & 02 & 04 & 0,72 \\
\hline Apelo Visual & 0 & 02 & 02 & 03 & 03 & 0,67 \\
\hline IVC2 & & & & & & $\mathbf{0 , 7 0}$ \\
\hline \multicolumn{7}{|l|}{ INFORMAÇÕES } \\
\hline Precisão da descrição & 0 & 0 & 0 & 01 & 04 & 0,95 \\
\hline $\begin{array}{l}\text { Qualidade das } \\
\text { informações }\end{array}$ & 0 & 0 & 0 & 03 & 02 & 0,85 \\
\hline $\begin{array}{l}\text { Quantidade de } \\
\text { informações }\end{array}$ & 0 & 0 & 0 & 03 & 02 & 0,85 \\
\hline Informações visuais & 0 & 02 & 0 & 01 & 02 & 0,85 \\
\hline IVC2 & & & & & & $\mathbf{0 , 8 7}$ \\
\hline
\end{tabular}

A idade média do público-alvo foi de 16,2 anos, sendo $60 \%$ do sexo feminino e $40 \%$ do sexo masculino. Os alunos do $1^{\circ}$ ano do Ensino Médio tiveram maior representação. A Tabela 2 exibe os resultados da avaliação do Teensaúde® pelo públicoalvo, para às dimensões: envolvimento, funcionalidade, estética e informações. Para essa análise, também foi considerado como ideal uma taxa de IVC superior a 0,78 (POLIT; BECK, 2006).

A dimensão "Envolvimento" analisou os aspectos relacionados à entretenimento, interesse, personalização, interação e grupo alvo. Com base na avaliação, o aplicativo obteve IVC2 de 0,83, sugerindo que ele possui a capacidade de divertir, despertar o interesse, e encontra-se bem direcionado ao público. Entretanto, na avaliação individual 
dos itens, o requisito "Interação" e "Personalização" apresentaram valores abaixo da média esperada, ou seja, 0,70 e 0,72, respectivamente. A dimensão "Funcionalidade" apresentou o maior IVC2 na avaliação pelo público-alvo, assim como todos os itens que a compõem individualmente. No que se refere a dimensão "Estética", os itens "Layout" e "Apelo visual" alcançaram notas superiores ao recomendado, enquanto o item "Gráfico" apresentou IVC1 um pouco abaixo do ideal $(0,75)$. Apesar disso, o IVC2 atingiu o valor de 0,78 , sugerindo que o aplicativo apresentou estética adequada para o público-alvo. Na dimensão "Informações" o IVC2 de 0,84 indicou que o aplicativo foi adequado ao seu objetivo e utilizou uma linguagem clara para os adolescentes sobre as temáticas abordadas. Na avaliação individual, todos os itens apresentaram IVC superior ao recomendado.

Tabela 2- Resultados da avaliação do aplicativo Teensaúde® pelo público-alvo.

\begin{tabular}{|c|c|c|c|c|c|c|}
\hline Análise & $\begin{array}{l}\text { Inade- } \\
\text { quado }\end{array}$ & $\begin{array}{l}\text { Insufi- } \\
\text { ciente }\end{array}$ & $\begin{array}{l}\text { Acei- } \\
\text { tável }\end{array}$ & $\begin{array}{l}\text { Muito } \\
\text { Bom }\end{array}$ & Exce-lente & IVC1 \\
\hline \multicolumn{7}{|l|}{ ENVOLVIMENTO } \\
\hline Entretenimento & 0 & 0 & 0 & 04 & 06 & 0,90 \\
\hline Interesse & 0 & 0 & 0 & 03 & 07 & 0,92 \\
\hline Personalização & 0 & 0 & 04 & 03 & 03 & 0.72 \\
\hline Interação & 0 & 02 & 01 & 04 & 03 & 0,70 \\
\hline Grupo Alvo & 0 & 0 & 01 & 01 & 08 & 0,92 \\
\hline IVC2 & & & & & & $\mathbf{0 , 8 3}$ \\
\hline \multicolumn{7}{|l|}{ FUNCIONALIDADE } \\
\hline Performance & 0 & 01 & 0 & 01 & 08 & 0,90 \\
\hline Facilidade de acesso & 0 & 0 & 01 & 01 & 08 & 0,92 \\
\hline Navegação & 0 & 01 & 0 & 01 & 08 & 1,00 \\
\hline Designer gestual & 0 & 0 & 0 & 0 & 10 & 0,82 \\
\hline IVC2 & & & & & & $\mathbf{0 , 9 1}$ \\
\hline \multicolumn{7}{|l|}{ ESTÉTICA } \\
\hline Layout & 0 & 0 & 02 & 04 & 04 & 0,80 \\
\hline Gráficos & 0 & 0 & 01 & 08 & 01 & 0,75 \\
\hline Apelo Visual & 0 & 01 & 0 & 05 & 04 & 0,80 \\
\hline IVC2 & & & & & & $\mathbf{0 , 7 8}$ \\
\hline \multicolumn{7}{|l|}{ INFORMAÇÕES } \\
\hline Precisão da descrição & 0 & 0 & 01 & 03 & 06 & 0,97 \\
\hline $\begin{array}{l}\text { Qualidade das } \\
\text { informações }\end{array}$ & 0 & 0 & 02 & 01 & 07 & 0,87 \\
\hline $\begin{array}{l}\text { Quantidade de } \\
\text { informações }\end{array}$ & 0 & 0 & 02 & 04 & 04 & 0,8 \\
\hline Informações visuais & 0 & 0 & 0 & 06 & 04 & 0,85 \\
\hline IVC2 & & & & & & $\mathbf{0 , 8 7}$ \\
\hline
\end{tabular}

Fonte: o autor (2021)

$\mathrm{Na}$ tabela 3 encontram-se as avaliações gerais, por dimensão, realizadas por cada grupo de avaliadores. As características de funcionalidade e informação obtiveram ótimas médias de aprovação, demonstrando que o aplicativo é agradável e realizou as atividades propostas de forma clara e prática, dentro de um nível de desempenho adequado. Vários estudos têm demonstrado uma maior preocupação dos desenvolvedores com a praticidade e facilidade em utilizar aplicativos (MIDDELWEERD et al., 2015). Por outro lado, os quesitos estética e envolvimento apresentaram uma média de aprovação um pouco abaixo 
das dimensões anteriores. Isso pode ter ocorrido, segundo os especialistas, pela quantidade de texto existente, poucas ferramentas interativas disponíveis, fonte e plano de fundo utilizados, que podem afastar o interesse do adolescente pelo aplicativo. Segundo Khan (2008), o envolvimento leva em consideração o quão divertido, interessante, customizável e interativo é o aplicativo, características que podem ser determinantes na escolha de um aplicativo, afinal, os celulares estão cada vez mais atrativos e populares. Ao calcular o IVC global dos especialistas e do público-alvo, foram observados os valores de 0,78 e 0,85 , respectivamente, que tornaram possível a validação do aplicativo Teensaúde®. Lopes et al. (2012) destacam que essa etapa se torna importante pelo fato de ser, a partir dessa avaliação, que podemos tornar aplicável à realidade o que está se produzindo. Assim, pretende-se avaliar a efetividade do Teensaúde ${ }^{\circledR}$ na promoção de conhecimentos sobre saúde e no incentivo do autocuidado em adolescentes do Ensino Médio de Escolas Estaduais de Juazeiro, Bahia.

Tabela 3- Avaliação do IVC global do aplicativo Teensaúde® pelos especialistas e pelo público-alvo

\begin{tabular}{lcc}
\hline \multicolumn{1}{c}{ DIMENSÕES } & IVC 2 & Público-alvo \\
\hline Envolvimento & 0,64 & IVC 2 \\
\hline Funcionalidade & 0,90 & 0,83 \\
\hline Estética & 0,70 & 0,93 \\
\hline Informações & 0,87 & 0,78 \\
\hline IVC Global & $\mathbf{0 , 7 8}$ & 0,87 \\
\hline
\end{tabular}

Fonte: o autor (2021)

\section{Conclusões}

O aplicativo Teensaúde® apresenta informações relevantes para a saúde do adolescente, dentro dos padrões tecnológicos de qualidade, com linguagem simples e acessível, uso de imagens pertinentes e ferramentas interativas e atraentes.

A avaliação dos especialistas e público-alvo e, principalmente, a aceitação pelo público-alvo demonstraram seu potencial de uso como um instrumento educacional em saúde direcionado aos adolescentes, por contribuí para a compreensão dessa temática e estimula o processo de autocuidado,

O aplicativo Teensaúde ${ }^{\circledR}$ poderá ser utilizado como ferramenta de apoio por profissionais das área da saúde e educação.

\section{Referências}

ALEXANDRE, N.M.C.; COLUCI, M.Z.O. Validade de conteúdo nos processos de construção e adaptação de instrumentos de medidas. Ciência \& Saúde Coletiva, v.16, n.7, p. 3061-3068, 2011.

BARRA, D.C.C.; PAIM, S.M.S.; DAL SASSO, G.T.M.; COLLA, G.W. Métodos para desenvolvimento de aplicativos móveis em saúde: revisão integrativa da literatura. Texto e Contexto de Enfermagem, v.26, n.4, p.e 2260017, 2017.

CABELlO, P.; CLARO, M.; CABELLO-HUTT, T. Mediação parental no uso de TIC segundo a percepção de crianças e adolescentes brasileiros: Reflexões com base na pesquisa TIC Kids Online Brasil 2014. In: CGI.br, Pesquisa sobre o uso da Internet por crianças e adolescentes no Brasil: TIC Kids Online Brasil 2016. São Paulo: CGI.br. CÁNOVAS, G. Cariño, he conectado a los niños. Bilbao: Ediciones Mensajero; 2015. 
CARR, N. A geração superficial: o que a internet está fazendo com os nossos cérebros.

Rio de Janeiro: Agir, 2011. 312 p.

CGI - CETIC: Comitê Gestor da Internet e Centro Regional de Estudos para o Desenvolvimento da Sociedade de Informação: Pesquisa TIC KIDS ONLINE - Brasil, 2015. Disponível em: https://cetic.br/pesquisa/kids-online/. Acesso em: 10 jun. 2019. FEHRING, R.J. Methods to validate nursing diagnoses. Saint Louis: Heart and Lung, v.16, n.6, p. 625-29, 1987.

FERREIRA, D.P.; GOMES JUNIOR, S.C.S. Aplicativos móveis desenvolvidos para crianças e adolescentes que vivem com doenças crônicas: uma revisão integrativa. Interface, v.25, p.1-17, 2021.

KHAN, M.M. Adverse effects of excessive mobile phone use. International Journal of Occupational and Environmental Health, v.21, n.2, p.89-93,2008.

LÉVY, P. Cibercultura. São Paulo: Editora 34; 2010.

LIVINGSTONE, S.; DAVIDSON, J.; BRYCE, J.; BATOOL, S.; HAUGHTON, C.; NANDI, A. Children's online activities, risks and safety: a literature review by the UKCCIS evidence group. London, UK: LSE, 2017.

LIVINGSTONE, S.; CARR, J.; BYRNE, J. One in three: Internet governance and children's rights. Innocenti Discussion Paper 2016-01. Florence, IT: UNICEF Office of Research, 2016.

LOPES, M.V.O.; SILVA, V.M.; ARAUJO, T.L. Methods for stablishing the accuracy of clinical indicator in predicting nursing diagnoses. International Journal of Nursing Knowledge, v.23, n.3, p. 134-39, 2012.

MALTA, D.C.; SARDINHA, L.M.V.; MENDES, I.; BARRETO, S.M.; GIATTI, L.; CASTRO. I.R.R.; MOURA, l.; DIAS, A.J.R.; CRESPO, C. Prevalência de fatores de risco e proteção de doenças crônicas não transmissíveis em adolescentes: resultados da Pesquisa Nacional de Saúde do Escolar (PENSE), Brasil, 2009. Revista Ciência \& Saúde Coletiva, v.15, supl.2, p.3009-19, 2010.

MANO, S.M.F. Ambiente virtual como facilitador do diálogo sobre sexualidade entre adolescentes: desenvolvimento e avaliação de um multimídia educativo. 2008. 170f. Tese (Doutorado em Ensino de Biociências e Saúde) Instituto Oswaldo Cruz, Rio de Janeiro.

MARQUES, R.A.F; GALLÃO, M.I. Desenvolvimento e validação do aplicativo Android RAbiomas. RENOTE, v.1, n.1, 2020.

MARTINS, R.B. O uso e desenvolvimento de jogos digitais educativos no Instituto Federal Baiano: uma experiência no campus Valença. 107. 110f. Dissertação (Mestrado em Profissional em Ciência da Computação) Universidade Federal de Pernambuco, Recife.

MIDDELWEERD, A.; VAN DER LAAN, D.; VAN STRALEN, M.M.; MOLLEE, J. What features do Dutch university students prefer in a smartphone application for promotion of physical activity? A qualitative approach. International Journal of Behavioral Nutrition and Physical Activity, v.12, n.1, p.1-11, 2015.

NIC.br. Núcleo de Informação e Coordenação do Ponto BR. 2019. Pesquisa sobre o uso da Internet por crianças e adolescentes no Brasil - TIC Kids Online Brasil 2018. Disponível em: https://cetic.br/pt/tics/kidsonline/2018/criancas/A1C/. Acesso em: 08 dez. 2021.

PASQUALI, L. Instrumentos Psicológicos: manual prático de avaliação. Brasília: LabPam/IBAP,1999.

PINTO, A.C.S.; SCOPACASA, L.F.; BEZERRA, L.L.A.L.; PEDROSA, J.V.; PINHEIRO, P.N.C. Uso de tecnologias da informação e comunicação na educação em 
saúde de adolescentes: revisão integrativa. Revista de Enfermagem UFPE, v.11, n.2, p.634-44, 2017.

POLIT, D.F.; BECK, C.T. Fundamentos de pesquisa em enfermagem: Avaliação de evidências para as práticas de enfermagem. 7.ed. Porto Alegre: Artmed, 2011.

POLIT, D.F.; BECK, C.T. The content validity index: are you sure you know what's being reported? Critique and recommendations. Research in Nursing \& Health, v.29, p. 489-97, 2006.

PREVITALE, A.P. A importância do brincar. Campinas. Monografia [Graduação] Universidade Estadual de Campinas, 2006.

ROSEN, L.D.; WHALING, K.; RAB, S.; CARRIER, L.M.; CHEEVER, N.A. Is Facebook creating "iDisorders"? The link between clinical symptoms of psychiatric disorders and technology use, attitudes and anxiety. Computers in Human Behavior, v.29, n.3, p.1243-54, 2012.

ROSSI, G. Um método orientado a objetos para o projeto de aplicações hipermídia. Tese (Doutorado), Pontifícia Universidade Católica do Rio de Janeiro, 1996.

SILVA, T.O.; SILVA, L.T.G. Os impactos sociais, cognitivos e afetivos sobre a geração de adolescentes conectados às tecnologias digitais. Revista da Associação Brasileira de Psicopedagogia, v.34, n.103, p.87-97, 2017.

SILVA, R.S. ANATOMIA-RA: aplicativo para android destinado ao ensino dos sistemas do corpo humano com a utilização de realidade aumentada. 2014. $101 \mathrm{f}$. TCC (Graduação) - Curso de Licenciatura em Computação, Universidade Estadual da Paraíba, Campina Grande, 2014.

SOARES, S.M.; AMARAL, M.A.; SILVA, L.B.; SILVA, P.A.B. Oficinas sobre sexualidade na adolescência: revelando vozes, desvelando pontos de vista dos alunos dos olhares médios de ensino. Escola Anna Nery Revista de Enfermagem, v.12, n.3, p.485491,2008.

SOUZA, K. Impactos do uso das redes sociais virtuais na saúde mental dos adolescentes: uma revisão sistemática da literatura. Educação, Psicologia e Interfaces, v.3, n.3, p.204217, 2019.

SOUZA, M. O real conceito de nativos e imigrantes digitais nas redes sociais digitais: conceitos, vivências e comportamento. 2013. 117 f. Dissertação (Mestrado em Redes Sociais), Universidade Estadual do Norte Fluminense Darcy Ribeiro, Rio de Janeiro.

STOYANOV, S.R.; HIDES, L.; KAVANAGH, D.J.; ZELENKO, O.; TJONDRONEGORO, D.; MANI, M. Mobile App Rating Scale: A New Tool for Assessing the Quality of Health Mobile Apps. JMIR Mhealth and Uhealth, v.3, p.1, p.01-09, 2015.

TORRES, R.A.M.; SILVA, M.A.M.; BEZERRA, A.E.M.; ABREU, L.D.P.; MENDONÇA, G.M.M. Comunicação em saúde: uso de uma web rádio com escolares. Journal of Health Informatics, v.7, n.2, p. 58-61, 2015.

VENTOLA, L.C. Mobile Devices and Apps for Health Care Professionals: Uses and Benefits. P \& T, v.39, n.5, p.356-364, 2014.

WILKINSON, A.S.; MILLER, Y.D. Improving health behaviors during pregnancy: A new direction for the pregnancy hand heald record. Journal of Obstetrics and Gynaecology, v.47, n.6, p.464-67, 2007.

ZAMBALDE, A.L., ALVES, R.M.; LOPES, M.A. Modelagem, autoria e análise de usabilidade de aplicação hipermídia direcionada ao setor agropecuário. UFLA, 1999. 